Review

\title{
miR-340: A multifunctional role in human malignant diseases
}

\author{
Zheng Huang ${ }^{1,2}$, Yesha $\mathrm{Xu}^{1}$, Maoping Wan ${ }^{1}$, Xixi Zeng ${ }^{1}$, Jianmin $\mathrm{Wu}^{1}{ }^{\bowtie}$ \\ 1. Institute of Genomic Medicine, Wenzhou Medical University, Wenzhou 325035, Zhejiang, P.R. China. \\ 2. Department of Anesthesia and Intensive Care, The Chinese University of Hong Kong, Hong Kong Special Administrative Region, P.R. China. \\ $\triangle$ Corresponding author: Jianmin Wu, Institute of Genomic Medicine, Wenzhou Medical University, Cha Shan University Town, No.1 Central North Road, \\ Wenzhou 325035, Zhejiang, P.R. China; Tel: +86 0577-88830096, Fax: +86 0577-88831359, E-mail: jianminwu81@hotmail.com; or, jmwu@wmu.edu.cn.
}

(1) The author(s). This is an open access article distributed under the terms of the Creative Commons Attribution License (https://creativecommons.org/licenses/by/4.0/). See http://ivyspring.com/terms for full terms and conditions.

Received: 2020.07.26; Accepted: 2020.11.09; Published: 2021.01.01

\begin{abstract}
MicroRNAs (miRNAs) are a class of short non-coding RNAs of approximately 22 nucleotides in length, which function by binding to the 3' UTR sequences of their target mRNAs. It has been reported that dysregulated miRNAs play pivotal roles in numerous diseases, including cancers, such as gastric, breast, colorectal, ovarian, and other cancers. Recent research efforts have been devoted to translating these basic discoveries into clinical applications that could improve the therapeutic outcome in patients with cancer. Early studies have shown that miR-340 may act either as an oncogene or a tumor suppressor by targeting genes related to proliferation, apoptosis, and metastasis, as well as those associated with diagnosis, treatment, chemoresistance, and prognosis. miR-340 has been shown to have a role in other diseases, such as autoimmune diseases, acute stroke, and alcoholic steatohepatitis. Nevertheless, the roles of miR-340 in human malignancies are still unclear, and the associated mechanisms are complex, involving a variety of signaling pathways, such as $\mathrm{Wnt} / \mathrm{\beta}$-catenin and the JAK-STAT pathways. Herein, we review the crucial roles of miR-340 in human cancers through the analysis of the latest research studies, with the aim of clarifying miR-340 function in malignant disease diagnosis, treatment, and prognosis, and to propose further investigations.
\end{abstract}

Key words: microRNAs, miR-340, malignant diseases, cancers, drug resistance

\section{Introduction}

Cancer is a major public health problem worldwide. The GLOBOCAN 2018 estimated that there would be 18.1 million new cases and 9.6 million deaths from cancer in 2018 [1]. Siegel et al. reported that there might be $1,762,450$ new cancer cases and 606,880 cancer-related deaths projected to occur in the United States in 2019 [2]. In China, Chen et al. predicted that there would be about 4,292,000 newly diagnosed invasive cancer cases in 2015, based on the data collected from 72 cancer registries [3]. Therefore, it is imperative to investigate the correlation between cancers and their respective risk factors; it is especially important to understand the molecular mechanisms of cancers, which may contribute to developing novel and effective pharmaceuticals and treatments.

MicroRNAs (miRNAs) are small non-coding RNAs of about 22 nucleotides in length. The mature
miRNA is loaded into the RNA-induced silencing complex (RISC), which is directed to target mRNAs, leading to translational repression and target mRNA degradation [4]. Recently, numerous studies have reported the roles for miRNAs in several human malignant diseases, especially cancers. miRNAs can function as modulators in multiple biological and pathological processes, such as cancer cell differentiation, proliferation, and apoptosis [5]. They can be used in cancer monitoring and therapy, and even in the clinical assessment of cancer patient outcomes [6]. For instance, Wang et al. have revealed that serum exosomal miR-17-5p, miR-130a-3p, and miR-93a-5 $p$ were downregulated and associated with breast cancer (BC) recurrence or distant organ metastasis [7]. Conversely, several upregulated miRNAs promoted tumor development and 
malignancy in ovarian cancer (OC) and intrahepatic cholangiocarcinoma [8, 9]. Moreover, miRNAs functioned as vital modulators in radiation-induced aggressive tumor behavior in human carcinoma cells [10]. These findings have led to the clinical trials of miR-34 replacement therapy (NCT01829971; terminated due to severe immune-related adverse events) and miR-122-based therapy LNA-antimiR-122 (SPC3649; successfully undergoing phase II trials) in cancer patients $[11,12]$. Thus, we believe that miRNAbased therapy may be a novel and promising method with which to treat various tumors in the future, when the roles and mechanisms of miRNAs in cancer are clearly understood.

Human miR-340 is an intragenic miRNA, located in the intronic region of the host gene RNF130, on chromosome $5 q 35.3$ [13]. miR-340 is highly conserved among mammals, and its expression pattern is similar to that of the host gene. One study showed that miR$340-5 p$ expression correlated with that of RNF130 in myeloma cell lines, and the expression of miR-340-5p was regulated by promoter hypermethylation of RNF130 [13]. The clustered H3K27Ac and highconfidence regulatory elements have been identified in the upstream region of miR-340, suggesting that the miR-340 locus was actively transcribed and intricately regulated (Fig. 1). miR-340 has been shown to participate in the progression of several diseases. Serum miR-340-5p levels were shown to be dysregulated in pulmonary sarcoidosis with Lofgren's syndrome [14], and serum miR-340-3p acted as a genetic biomarker associated with human longevity [15]. miR-340-5p was involved not only in human heart failure and pathological cardiac hypertrophy [16], but also in osteoclast formation and osteoporosis progression [17]. Recently, a number of studies have reported that miR-340 plays a critical role in tumor initiation and progression, by targeting multiple oncogenes such as SKP2, FHL2, c-Met, and ROCK1 (Table 1). Some signaling pathways related to tumorigenesis, such as AKT [18], SOCS3/JAK-STAT [19], and Wnt/ $\beta$-catenin pathways [20], were shown to be suppressed by miR-340.

In this review, we present and synthesize the roles and mechanisms of action of miR-340 in cancer cell proliferation, apoptosis, invasion and metastasis, drug resistance, and cancer diagnosis, as well as patient survival and prognosis, aiming to provide a significant foundation for clinical applications and future investigations.

\section{Biogenesis and expression regulation of miR-340}

Previous reports have demonstrated that the gene promoter region, which is distal to the transcription start site, contains uniformly dense CpG islands. These islands were methylated in cancer cells, but not in normal cells [21]. Transcriptional silencing via $\mathrm{CpG}$ methylation in the promoter is an important regulatory mechanism for the downregulation of miRNAs, which may promote tumor initiation and development [20, 22-25]. Recently, hypermethylation of CpG islands in the miR-340 gene promoter region has been found in gastric cancer (GC) [26]. Our recent study revealed that treatment with 5-aza-2'deoxycytidine or TSA restored the levels of miR-340 in OC cells [20]. These results implied that the silencing of miR-340 expression was mediated through the $\mathrm{CpG}$ methylation of the upstream regions in the miR-340 promoter.

Table 1. miR-340 Dysregulates in Various Cancers and Their Target Genes

\begin{tabular}{|c|c|c|}
\hline Cancer Type & Target gene & Reference \\
\hline \multicolumn{3}{|l|}{ miR-340 Downregulation } \\
\hline BC & LGR5 & [56] \\
\hline BC & -- & {$[7,103]$} \\
\hline BC & ZEB1 & [29] \\
\hline$B C$ & CTNNB1, c-MYC & [71] \\
\hline $\mathrm{BC}$ & c-Met & [70] \\
\hline CRC & ANXA3 & [99] \\
\hline CRC & RLIP76 & [50] \\
\hline CRC & -- & [78] \\
\hline Gallbladder carcinoma & NT5E & [79] \\
\hline GBM & -- & {$[91,92,104]$} \\
\hline GBM & NRAS & [95] \\
\hline GBM & CDK6, cyclin-D1, cyclin-D2 & [42] \\
\hline GBM & Bcl-w, Sox2 & [53] \\
\hline GBM & ROCK1 & [52] \\
\hline GC & SPP1 & [59] \\
\hline GC & -- & [18] \\
\hline $\mathrm{HCC}$ & DcR3 & [112] \\
\hline $\mathrm{HCC}$ & SKP2 & [47] \\
\hline $\mathrm{HCC}$ & JAK1 & [48] \\
\hline $\mathrm{HCC}$ & Nrf2 & [87] \\
\hline Laryngeal squamous cell carcinoma & EZH2 & [46] \\
\hline Melanoma & -- & {$[34,113]$} \\
\hline NSCLC & ZNF503 & [114] \\
\hline NSCLC & CDK4 & [40] \\
\hline NSCLC & SKP2 & [44] \\
\hline OC & FHL2 & [20] \\
\hline OC & BAG3 & [67] \\
\hline OC & NF-кB1 & [66] \\
\hline OS & STAT3 & [115] \\
\hline OS & LPAAT $\beta$ & [86] \\
\hline OS & ROCK1 & {$[77,101]$} \\
\hline Oral Squamous Cell Carcinoma & Glut1 & [116] \\
\hline Pancreatic cancer & BICD2 & [117] \\
\hline $\mathrm{PCa}$ & MDM2 & [57] \\
\hline $\begin{array}{l}\text { Triple-negative breast cancer } \\
\text { miR-340 Upregulation }\end{array}$ & -- & [118] \\
\hline GC & SOCS3 & [19] \\
\hline GC & cyclin G2 & [41] \\
\hline GC & -- & {$[93,107]$} \\
\hline Thyroid cancer & BMP4 & [58] \\
\hline
\end{tabular}

BC: breast cancer; CRC: colorectal cancer; GBM: glioblastoma multiforme; GC: gastric cancer; HCC: hepatocellular carcinoma; NSCLC: non-small cell lung cancer; OC: ovarian cancer; OS: osteosarcoma; PCa: Prostate cancer. 


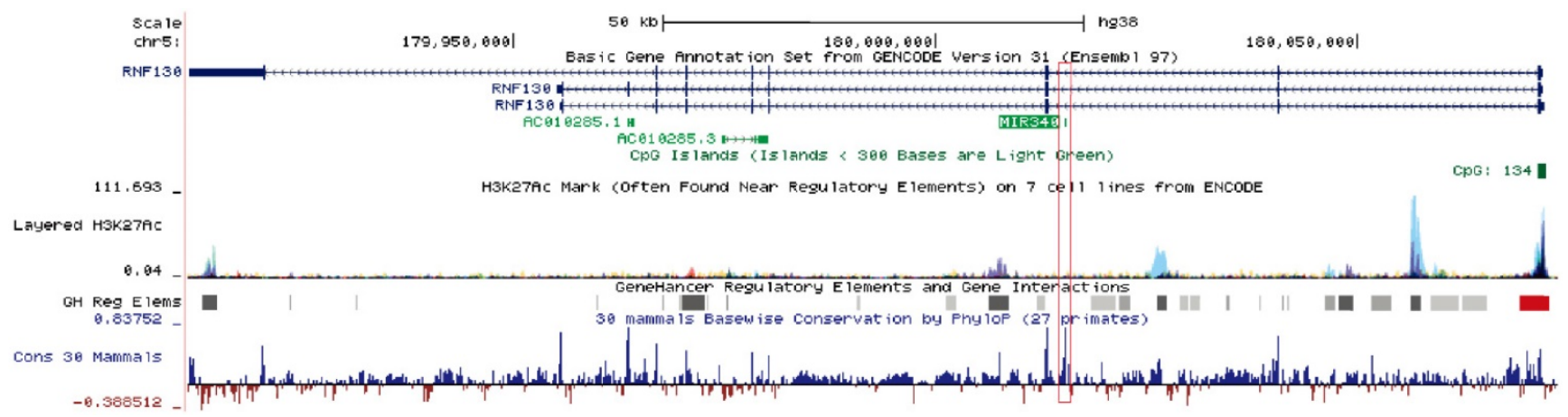

Figure 1. Schematic diagram of the miR-340 locus. miR-340 is an intragenic miRNA located in the intronic region of the host gene RNFI30. It has similar expression patterns as those of the host gene. In the upstream regulatory regions as well as the gene body, an active epigenetic marker, the H3K27Ac cluster, was identified by the ENCODE project; a high-confidence enhancer/promoter cluster was identified by the GeneHancer project. The information was obtained from the UCSC Genome Browser.

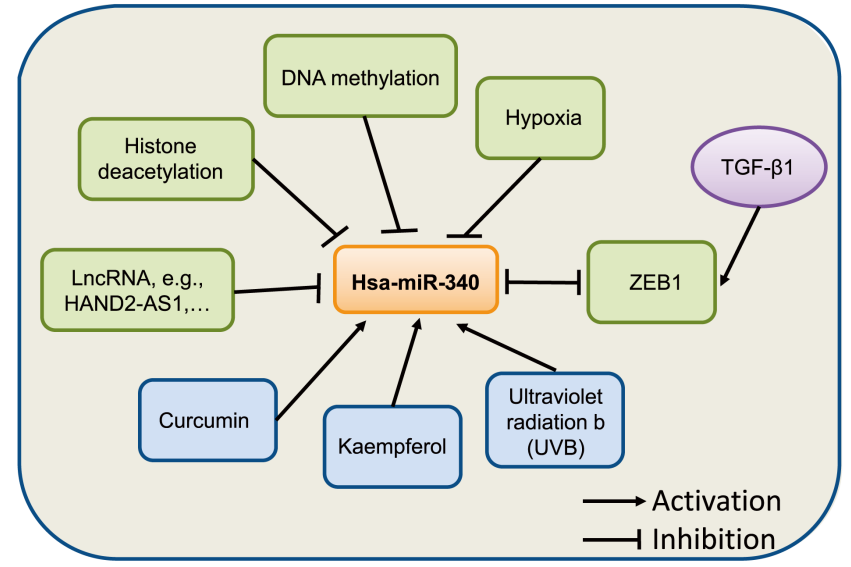

Figure 2. Summary of miR-340 expression regulation. miR-340 expression is modulated by various factors, such as epigenetic modification, transcription factors, and hypoxia.

Transcription factors act as regulators that modulate miRNA expression in many cancers. For example, a decrease in SIRT1 stimulates the expression of p53, which leads to the activation of miR-34a [27, 28]. Similarly, zinc finger E-box-binding homeobox 1 (ZEB1) acts as a transcription factor that suppresses miR-340 expression in BC [29]. Hypoxia is a common microenvironment in multi-pathophysiologic progression, including tumorigenesis [30, 31]. There is increasing evidence that miRNAs are involved in tumorigenesis and drug resistance driven by hypoxia [32, 33]. miR-340-5p was identified as being significantly downregulated by hypoxia in melanoma cells [34]. Du et al. found that the antioxidant ferulic acid could promote hypoxia signaling by inducing hypoxic-induced factor, which suppressed miR-340-5p promoter activation through hypoxia response element (HRE) motifs [35].

The natural compound curcumin has also been shown to induce the expression of miR-340 in human pancreatic cancer cells. miR-340 was identified as being significantly upregulated following curcumin treatment [36]. A recent study showed that miR-340 is regulated by nanocurcumin in relapsing-remitting multiple sclerosis [37]. Another compound, Kaempferol, was found to upregulate miR-340 expression in human lung cancer cells [38]. In retinal pigment epithelium (RPE) cells [39], UVB irradiation induced the expression of miR-340, which promoted RPE cell apoptosis and suppressed cell viability by affecting p53, p21, and caspase-3 protein expression. Collectively, these evidences suggest that the expression of miR-340 can be regulated by epigenetic modification, transcriptional regulation, and other factors, although further studies on the mechanism underlying miR-340 dysregulation in human cancer are needed (Fig. 2).

\section{Functional roles of miR-340 in cancers}

\section{Role of miR-340 in cell proliferation in cancer}

Increasing evidence has indicated that miR-340 is associated with various genes mediating cancer cell proliferation. Cyclin D/cyclin G2 and CDK4/6 have been shown to be upregulated in a variety of human cancer cells [40-42]. The p27/p21 gene, which is a cell cycle inhibitor, binds to and prevents the activation of cyclin E-CDK2 or cyclin D-CDK4/6 complexes, and thus controls cell cycle progression at the G1 phase, which is known to be involved in cancers [43]. miR-340 was shown to induce the accumulation of p27 and subsequent cell cycle arrest by targeting three negative regulators of p27: PUM1, PUM2, and SKP2, indicating that miR-340 could repress non-small cell lung cancer (NSCLC) cell proliferation [44]. miR-340 also inhibits lung cancer cell proliferation by targeting CDK4 [40]; while it has been reported that CDK4 is involved in accelerating NSCLC cancer procession and combined inhibition of CDK4 could be effective to treat NSCLC [45]. Additionally, transfection of miR-340 or silencing of EZH2 has been shown to impede laryngeal squamous cell carcinoma progression by inducing p27 expression and suppressing PI3K/AKT activation [46]. 


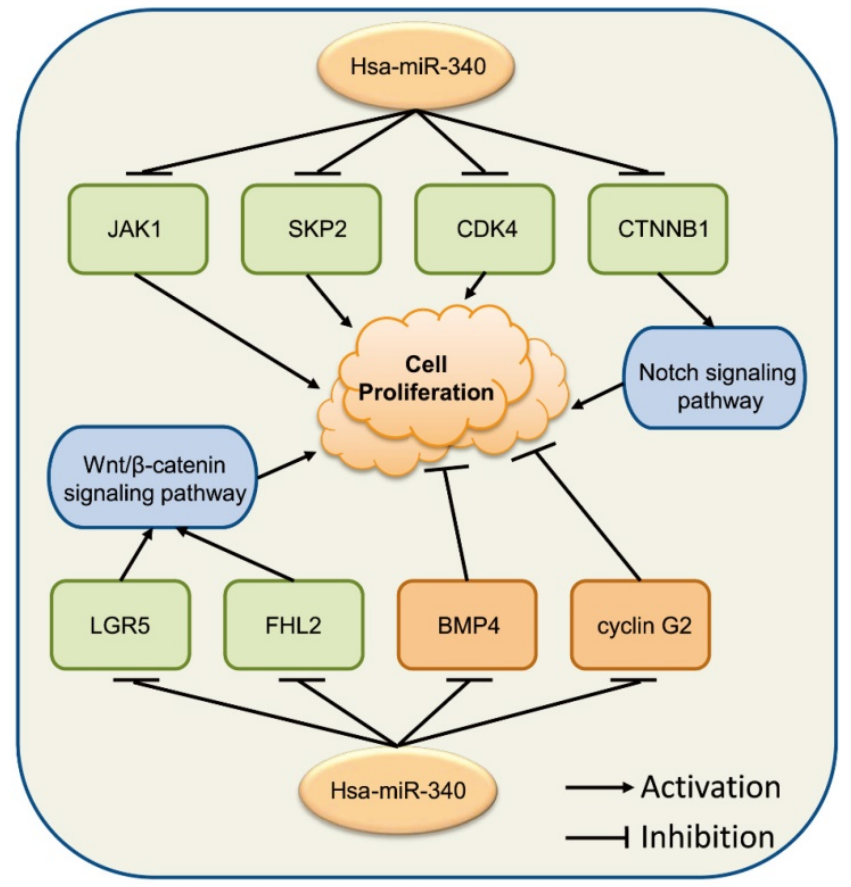

Figure 3. miR-340 is associated with various genes modulating cancer cell proliferation. Upregulated miR-340 inhibits the expression of JAKI, SKP2, and CDK4, repressing cell proliferation, while it inhibits the expression of BMP4 and cyclin G2, inducing cell proliferation. miR-340 inhibits the $\mathrm{Wnt} / \beta$-catenin signaling pathway by targeting LGR5 or FHL2, as well as the CTNNB1-mediated Notch signaling pathway, resulting in repressed cell proliferation.

In hepatocellular carcinoma (HCC), miR-340 inhibits cell proliferation and tumor growth by inhibiting SKP2 expression [47], and regulating the JAK1/STAT3 pathway [48]. Additionally, miR-340 also functions as a tumor suppressor in colorectal cancer (CRC) by regulating the alternative splicing of the PKM gene [49] or directly targeting RLIP76 [50]. Restoration of miR-340 expression in angiosarcoma cells reduced cell proliferation by negatively regulating SIRT7 expression [51]. In addition, miR-340 expression was discovered to be downregulated in both glioma cell lines and tissues. Increasing miR-340 levels dramatically inhibited glioma cell proliferation, and induced cell-cycle arrest via inhibition of the target gene ROCK1, as well as several oncogenes, including p-AKT, EZH2, EGFR, BMI1, and XIAP [52]. miR-340 was found to specifically target the $3^{\prime}$ UTRs of CDK6, cyclin-D1, and cyclin-D2, leading to the arrest of glioblastoma multiforme (GBM) cells in the G0/G1 cell cycle phase [42]. Furthermore, miR-340 was shown to be a potential therapeutic agent for GBM via its inhibitory effects on Bcl-w-induced platelet-derived growth factor-A (PDGF-A) and Sox2 activation [53]. A recent study revealed that miR-340 could suppress osteosarcoma (OS) cell proliferation by inactivating the Notch signaling pathway by down-regulating CTNNB1 [54]. It has been reported that the overexpression of miR-340 inhibited human esophageal squamous cell carcinoma (ESCC) cell growth by modulating the expression of phosphoserine aminotransferase 1 (PSAT1), and it may contribute to the progression of ESCC [55]. Additionally, miR-340 inhibited BC cell proliferation by targeting the expression of ZEB1 or LGR5 through the Wnt/ $\beta$-catenin pathway, which might provide a new perspective for $B C$ treatment $[29,56]$. miR-340 functioned as a tumor suppressor in prostate cancer (PCa) through the MDM2-p53 pathway by directly targeting MDM2 [57].

Nevertheless, Xiao and colleagues found that the level of miR-340 was significantly higher in MKN-28 cells than in GES-1 cells; anti-miR-340 attenuated cell proliferation and arrested cell cycle in MKN-28 cells by upregulating SOCS3 expression to suppress the JAK-STAT3 signaling pathway [19]. miR-340 was found to promote thyroid cancer growth in vitro and in vivo by inhibiting BMP4 [58]. The role of miR-340 is ambiguous in GC $[19,59]$, and needs to be further investigated.

These observations show that the relationship between miR-340 and cancer cell progression is complex (Fig. 3). miR-340 might perform different context-dependent roles in different cancer cells, acting as either a tumor growth suppressor or an oncogene, which requires further investigation to verify its exact roles in specific cancers.

\section{miR-340 and cell apoptosis}

Accumulating evidence suggests that the dysregulation of cell apoptosis is involved in a majority of diseases, as this process includes multitudes of classical signaling pathways and proteins. Death receptors, mitochondria, and caspase signaling pathways have been reported to participate in cancer modulation [60]. Bcl-2 and Bax are members of the Bcl-2 family, which has been implicated in the regulation of cell apoptosis [61]. Bcl-2 is an anti-apoptotic protein and can directly prevent cell apoptosis by limiting the pro-apoptosis member activity of the Bcl-2 family [62]. Bax is a proapoptotic member of the Bcl-2 family that regulates programmed cell death, and is associated with increased survival [63]. miR-340 increased the expression levels of BIM and Bax, but decreased those of Notch and Bcl-2, inducing OS cell apoptosis by inactivating the Notch signaling pathway via targeting CTNNB1 [54]. In CRC, miR-340 was observed to target RLIP76 [50] and REV3L [64] to mediate cell apoptosis. Moreover, miR-340 increased the levels of apoptosis-related factors pro-caspase 3, cleaved-caspase 3, and Bax, but inhibited Bcl-2 in SGC-7901 cells [18]. A similar phenomenon was also observed in the endometrial carcinoma cell line RL 95-2 [65]. In OC, miR-340 was shown to induce cell 
apoptosis by the downregulation of NF-kB1 [66]. Overexpression of miR-340 improved apoptosis in SKOV3 cells through the negative regulation of BAG3, which might be involved in the regulation of the PI3K/AKT pathway [67]. Altogether, these studies indicated that miR-340 exerts significant effects in cancer cell apoptosis (Fig. 4).

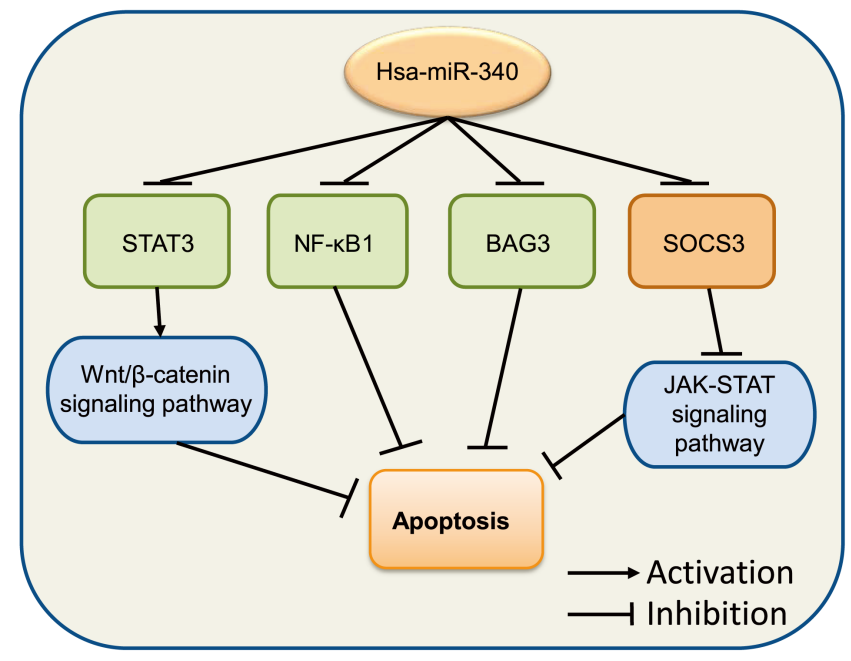

Figure 4. miR-340 is involved in the regulation of cancer cell apoptosis. Upregulated miR-340 inhibits NF-KB1, BAG3, and STATS as well as its downstream Wnt/ $\beta$-catenin signaling pathway, which induces cell apoptosis, while it inhibits the expression of SOCS3 but suppresses apoptosis by regulating the JAK-STAT signaling pathway.

\section{Role of miR-340 in cancer invasion and metastasis}

Cancer invasion and metastasis are usually happened in highly malignant cancer patients and involved in complex mechanism. The epithelialmesenchymal transition (EMT) is a key process in tumorigenesis, and Vimentin and E-cadherin are typical biomarkers of EMT [68]. Wu et al. performed microarray-based profiling analysis of miRNA expression in BC lines with different invasion capacity and found that miR-340 was significantly decreased in BC cell lines with high invasive potential $[69,70]$. Restoration of miR-340 in the BC cell line suppressed the expression of target genes, such as $c-$ Met [70], CTNNB1, c-MYC [71], and MYO10 (myosin X) [72] to inhibit cell invasion and metastasis via several signaling pathways. Transforming growth factor (TGF)- $\beta$ signaling is important for EMT and the expression of ZEB1 [73]; treatment with the TGF- $\beta 1$ resulted in increased levels of $Z E B 1$ expression, while decreasing the level of miR-340 in BC [29]. Overexpression of miR-340 inhibited the migration and invasion of cervical cancer cells by targeting EphA3 and adjusting the EMT pathway [74]. Infection with the hepatitis B virus (HBV) is a leading cause of hepatocellular carcinoma (HCC) [75], and HBV promotes the migration of liver cancer cells by downregulating miR-340-5p to induce STAT3 overexpression, indicating that STAT3 plays a key role in regulating cell migration in HBV-HCC involving miR-340-5p [76]. It has been observed that miR-340 suppressed tumor growth and metastasis in OS cells in vitro and in vivo by targeting ROCK1 [77]. Takeyama et al. found that miR-340 expression was significantly decreased in the $\operatorname{EpCAM}(+)$ bone marrow cells of CRC patients with liver metastasis, showing that miR-340 in the bone marrow might play an important role in regulating the metastasis cascade in CRC [78]. In gallbladder carcinoma, miR-340 targeted NT5E to function as a significant suppressor of tumor metastasis [79].

These series of findings indicate that miR-340 has vital functions in tumor invasion and metastasis (Fig. 5). Increasing the levels of miR-340 expression appears to repress cancer invasion and metastasis in most types of cancer. We hypothesize that these findings cover only a few functions of miR-340 in tumor invasion and metastasis. In addition, other targets and signaling pathways related to miR-340 might be included in further investigations.

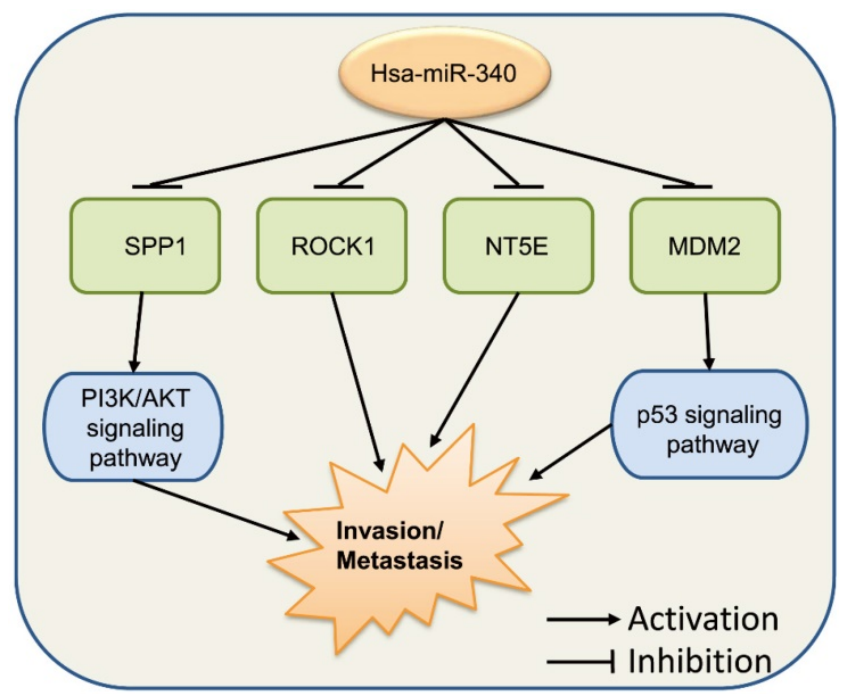

Figure 5. miR-340 functions in cancer invasion and metastasis. ROCKI, NT5E, SPPI, and MDM2 are the target genes of miR-340, and miR-340 inhibits their functions as well as the downstream PI3K/AKT and p53 signaling pathways, which results in the repressed cell invasion and metastasis.

\section{Mechanisms of drug resistance}

Chemotherapy is the first-line approach to the treatment of cancers, whereas it seems to result in drug resistance and lead to complications. Therefore, exploring novel, safe, and highly effective treatments is imperative [80, 81]. Cisplatin (CDDP) as a custom drug for treating cancers has usually been applied in clinical patients. However, decreased CDDP sensitivity in tumors becomes the biggest obstacle in cancer therapy [82]. Recently, several studies have 
shown that miRNAs were associated with cancer drug resistance; for instance, it was reported that miR-134 plays various roles in drug resistance in diverse cancers [83]. Furthermore, miR-128-3p was found to confer chemoresistance-associated metastasis in NSCLC [84]. Similarly, miR-340 was also involved in cancer drug resistance. Forced miR-340 expression in drug-resistant OS cells significantly reduced multidrug resistance-associated protein 1 and P-gp expression, and enhanced their sensitivity to CDDP by targeting ZEB1 and LPAAT $\beta[85,86]$. In HCC, miR-340 was significantly downregulated, whereas Nrf2 was upregulated in HepG2/ CDDP cells. Transfection of miR-340 mimicked the suppressed Nrf2-dependent antioxidant pathway, and enhanced the sensitivity of HepG2/CDDP cells to CDDP [87]. Further investigation uncovered that the NRAL/miR-340-5p/Nrf2 axis mediated CDDP resistance in HCC [88]. In addition, miR-340-5p was reported to be involved in trastuzumab resistance in HER2-positive BC cells [89]. Melanoma is usually highly refractory to chemotherapy, which is mainly due to the high heterogeneity and plasticity of melanoma cells that is strictly connected to changes in tumor microenvironment [34]. Wozniak et al. uncovered that the increased levels of ABCB5, a transmembrane transporter involved in drug resistance considered as a marker of melanoma stemlike cells, could be a result of a significant miR-340-5p downregulation [34].

Additionally, miR-340 was also involved in radiation-induced aggressive tumor formation [10]. It has been reported that interleukin-4 (IL-4) and IL-4Ra (IL-4 receptor) were highly expressed in various human cancer cells following radiation treatment. High expression of IL-4 in patients with cancer is strongly correlated with poor survival. It has been found that IL-4 expression was reduced by miR-340 and miR-429, which were in turn downregulated by ionizing radiation. This study presented a conceptual advance suggesting that combining radiotherapy with genetic therapy to inhibit IL-4 may be a promising strategy for preventing post-radiation recurrence and metastasis in patients [10]. Treatment with the natural compound curcumin or miR-340 induced pancreatic cancer cell apoptosis [36]. Curcumin could increase miR-340 expression and reduce the expression of the oncogene XIAP, which was identified as a direct target of miR-340. This interaction may provide the basis for novel treatment strategies for patients with pancreatic cancer [36].

These findings suggest that miR-340 plays various roles in cancer drug resistance. A combination of genetic therapy with chemotherapy or radiotherapy may be a useful strategy to overcome drug resistance; however, this approach needs further investigation to validate it before applying it to clinical therapy.

\section{Potential clinical applications of miR-340 in cancers}

\section{miR-340 serves as a promising biomarker}

Cancers have often progressed to middle and advanced stages at the time of diagnosis; therefore, early tumor and carcinoma screening and diagnosis are crucial. Evidence suggests that miRNAs are stable and could not be easily degraded in patient serum [90]. Therefore, miRNAs might be a class of novel biomarkers valuable for the detection of diseases. Pulmonary sarcoidosis is associated with dysregulated expression of intracellular miRNAs. Novosadova et al. revealed that the miR-340-5p levels were dysregulated in the serum of patients with LS, implying that miR-340 might play a diagnostic role [14]. In glioma patient serum [91, 92], miR-340 was found to be significantly elevated, and further bioinformatics analysis found that it possibly played important roles in the regulation of glioma signaling pathways, suggesting that miR-340 of the peripheral blood might serve as a new biomarker for glioma diagnosis, with high specificity and sensitivity. In the analysis of global miRNA expression profile in GC, miR-340 was found to be highly elevated in cancer cells, which suggests a potential role of miR-340 in the diagnosis of GC [93]. As we have detailed earlier, miR-340 was found to be dysregulated in a variety of human tumors (Table 1), which might make it a promising and qualified biomarker for cancer diagnosis.

\section{miR-340 in cancer patient survival and prognosis}

With the mechanisms of the function and regulation of miRNAs in cancer gradually revealed, miRNA-based treatment might be a possible candidate approach in the near future. It has been reported that miR-340 was notably downregulated in NSCLC tissues, and lower miR-340 expression was positively correlated with lymph node metastasis, larger tumor size, advanced TNM stage, and poor prognosis in NSCLC patients [40]. Therefore, miR-340 might be a promising tool for treating cancers or exploring mechanisms of disease progression. In BC, miR-340 was observed to be downregulated and loss of miR-340 expression was associated with shorter overall survival [70]. Recent studies have shown that exosomes were detected as an indicator for diagnosis and prognosis of BC in clinical settings. miR-340-5p was identified to be associated with tumor recurrence 
or distant organ metastasis in BC patients through its detection in patient peripheral blood exosomes, which can be used as prognostic biomarker in these patients $[7,94]$. GBM generally has a survival rate of 12 months from diagnosis. Further investigation demonstrated that miR-340 may thus represent a novel marker for GBM diagnosis and prognosis, and may be developed into a tool to improve the treatment of GBM [95]. Another study suggested that a miR-340-5p-macrophage feedback loop modulated the progression and tumor microenvironment of GBM, and may represent a prognostic biomarker [96].

DTCs in the bone marrow can be a sensitive marker for cancer spread from the primary tumor, which is associated with prognosis; EpCAM acts as a specific epithelial cell protein for detecting DTCs in the bone marrow in patients with CRC [97, 98]. miR-340 expression was significantly decreased in $\operatorname{EpCAM}(+)$ bone marrow cells in patients with liver metastasis. Survival analysis in 136 patients with CRC indicated that lower miR-340 expression was correlated with shorter five-year disease-free survival and poorer five-year overall survival [98]. In addition, the CRC group with low miR-340 and high c-Met expression had the worst prognosis [78]. Consistently, Yang et al. revealed that CRC patients with a low expression of miR-340-5p had a shorter overall survival and progression-free survival (PFS) than those with a high expression of miR-340-5p [99].

This part of the paper has focused upon the relationship between miR-340 and patient survival and prognosis in some cancers. We hope it provides evidence for further studies on cancer patient prognosis as well as disease burden. ROCK1, a protein serine/threonine kinase, was reported to function as a key modulator of cell motility, tumor cell invasion, and actin cytoskeleton organization [100]. Recently, ROCK1 was discovered to be a target of miR-340 and was dramatically upregulated in GBM tissues and cells. Survival in GBM patients with high levels of miR-340 was significantly extended in comparison to that in patients with low levels of miR-340, which suggested that miR-340 was a glioma killer and a potential prognostic biomarker and therapeutic target in GBM [52]. A similar relationship between miR-340 and ROCK1 was revealed in pediatric OS [101]. miR-340-low/ROCK1-high expression was significantly associated with both shortest overall survival and PFS. Further analysis suggested that the combined miR-340 downregulation and ROCK1 upregulation might be linked to tumor progression and adverse prognosis in pediatric OS [101]. Yin et al., however, found that the expression of miR-340 was significantly elevated in both GC tissues and cells. Patients with high expression of miR-340 had shorter overall survival and disease-free survival [41]. These data suggest that miR-340 may serve as a novel prognostic biomarker in GC. This conclusion is completely opposite to those of other investigations, whether there will be more analogous findings or not needs to be confirmed.

\section{Potential applications of miR-340 in cancer therapy}

As the mechanism and role of miRNAs in human diseases are gradually unraveled, recent studies have started exploring the role of miRNAs as therapeutic agents. miR-340 acts as a tumor suppressor in multiple types of cancers, and emerging evidence has shown that miR-340-based united therapy was a promising approach to cancer treatment [17, 102]. The intratumoral expression of miR-340 prior to neoadjuvant chemotherapy could be used to predict pathologic complete response ( $\mathrm{pCR}$ ) and a profile of miR-340high identified patients who were unlikely to achieve $\mathrm{pCR}$, and therefore might benefit from alternative treatment options, including earlier surgery [103]. This study identified miRNAs as promising predictive biomarkers, which could aid in optimization of BC management and treatment stratification [103]. Combined with high throughput sequencing analysis, miR-340 activity in cell proliferation, adhesion to the extracellular matrix, and tumor cell invasion, were identified in a tissue model, which was constructed by GBM developed into a stem cell-derived human engineered neural tissue, and also confirmed in GBM biopsies. miR-340 was a strong modulator of GBM aggressiveness and may constitute a therapeutic target for treatment of malignant gliomas [104]. Similarly, restoration of miR-340 levels dramatically inhibited glioma cell proliferation, induced cell-cycle arrest and apoptosis, suppressed cell motility, and promoted autophagy and terminal differentiation, indicating an important role of miR-340 as a glioma killer, and suggesting a potential prognosis biomarker and therapeutic target for GBM [52]. Takeyama et al. discovered that pre-miR-340 administration inhibited the growth of colon cancer cells and suppressed c-Met expression in vitro [78]. Further analysis demonstrated that systemic pre-miR-340 administration suppressed the growth of pre-established HCT116 tumors in animal therapeutic models. These findings indicated that miR-340 may be useful as a therapeutic tool to treat CRC [78]. Similarly, miR-340 may act as a potential new therapeutic target for the treatment of OS [77]. A recent study revealed that the overexpression of miR-340 promoted macrophages to acquire M1-like phenotype polarized in peripheral and tumor immune microenvironments and increased $\mathrm{T}$ cell 
levels, especially the $\mathrm{CD}^{+} \mathrm{T}$ cells, contributing to the antitumor effect of miR-340 on pancreatic ductal adenocarcinoma [105]. Taken together, our results indicate that miR-340 plays a role as an active agent against tumors, which merits further investigations for clinical development in cancer disease.

\section{Conclusions and future perspectives}

In this review, we presented some interesting findings that might be beneficial for clinical applications and future studies. miR-340 is dysregulated in various tumors and carcinomas, where it may function as either a tumor suppressor or an oncogene. For instance, miR- 340 was shown to be downregulated in two studies [18, 106], but was upregulated according to other work on GC $[41,107]$. Functionally, miR-340 was discovered to either repress or promote cancer cell proliferation and xenograft development and boost tumor cell apoptosis, migration, and metastasis, as well as benefit patient survival and prognosis. The molecular and modulated mechanisms of miRNA are extremely complicated and variable, and we found that miR-340 has diverse target genes and becomes involved in sophisticated signaling pathways when it functions in cancer $[19,56]$. There will be other targets and signaling pathways of miR-340 related to cancer, which might demand further investigations. Recently, long non-coding RNAs (lncRNAs) have emerged as crucial regulatory factors in diverse pathological processes, especially in tumorigenesis. Evidence has demonstrated that miR-340 was sponged by lncRNAs involved in tumor initiation and development [108-111], which may provide a new direction for an miR-340-based investigation of cancers.

Up to now, the role of miR-340 has been gradually illustrated in various cancers and other diseases. However, its functions in most malignant diseases are ambiguous. It might take a long time before it can be applied in clinical settings, similar to miR-34a or miR-122. However, encouragingly, increasing developing strategies are accelerating the investigation process, which brings us hope to overcome the present issues. Combined with current research results, in the future, the investigation of miR-340-based transgenic mice may be a promising direction to illustrate its functions more clearly under physiological conditions. In this paper, we summarized the role of dysregulation of miR-340 in a variety of malignant diseases, especially in cancers, highlighting the multiple roles of miR-340 in cancer initiation and progression.

\section{Abbreviations}

miRNAs: microRNAs; RISC: RNA-induced silencing complex; $\mathrm{BC}$ : breast cancer; $\mathrm{OC}$ : ovarian cancer; NSCLC: non-small cell lung cancer; OS: osteosarcoma; CRC: colorectal cancer; GC: gastric cancer; GIC: glioma-initiating cell; GBM: glioblastoma (multiforme); ROCK1: Rho-associated protein kinase 1; ZEB1: zinc finger E-box-binding homeobox 1; HRE: hypoxia response element; RPE: retinal pigment epithelium; HCC: hepatocellular carcinoma; PCa: prostate cancer; EMT: epithelial-mesenchymal transition; TGF: transforming growth factor; HBV: hepatitis B virus; DTC: disseminated tumor cell; CDDP: cisplatin; pCR: pathologic complete response; lncRNA: long non-coding RNA.

\section{Acknowledgements}

This work was supported by the National Natural Sciences Foundation of China (grant NSFC81472651) and Zhejiang Provincial Natural Sciences Foundation (LY20H160016). We would like to thank Editage (www.editage.cn) for English language editing.

\section{Competing Interests}

The authors have declared that no competing interest exists.

\section{References}

1. Bray F, Ferlay J, Soerjomataram I, Siegel RL, Torre LA, Jemal A. Global cancer statistics 2018: GLOBOCAN estimates of incidence and mortality worldwide for 36 cancers in 185 countries. CA Cancer J Clin. 2018; 68: 394-424.

2. Siegel RL, Miller KD, Jemal A. Cancer statistics, 2019. CA Cancer J Clin. 2019; 69: 7-34.

3. Chen W, Zheng R, Baade PD, Zhang S, Zeng H, Bray F, et al. Cancer statistics in China, 2015. CA Cancer J Clin. 2016; 66: 115-32.

4. Hammond SM. An overview of microRNAs. Adv Drug Deliv Rev. 2015; 87: 3-14.

5. Di Leva G, Garofalo M, Croce CM. MicroRNAs in cancer. Annu Rev Pathol. 2014; 9: 287-314.

6. Hayes J, Peruzzi PP, Lawler S. MicroRNAs in cancer: biomarkers, functions and therapy. Trends Mol Med. 2014; 20: 460-9.

7. Wang M, Ji S, Shao G, Zhang J, Zhao K, Wang Z, et al. Effect of exosome biomarkers for diagnosis and prognosis of breast cancer patients. Clinical \& translational oncology: official publication of the Federation of Spanish Oncology Societies and of the National Cancer Institute of Mexico. 2018; 20: 906-11.

8. Salem M, O'Brien JA, Bernaudo S, Shawer H, Ye G, Brkic J, et al. miR-590-3p Promotes Ovarian Cancer Growth and Metastasis via a Novel FOXA2-Versican Pathway. Cancer research. 2018; 78: 4175-90.

9. Li H, Zhou ZQ, Yang ZR, Tong DN, Guan J, Shi BJ, et al. MicroRNA-191 acts as a tumor promoter by modulating the TET1-p53 pathway in intrahepatic cholangiocarcinoma. Hepatology. 2017; 66: 136-51.

10. Kim ES, Choi YE, Hwang SJ, Han YH, Park MJ, Bae IH. IL-4, a direct target of miR-340/429, is involved in radiation-induced aggressive tumor behavior in human carcinoma cells. Oncotarget. 2016; 7: 86836-56.

11. Gebert LF, Rebhan MA, Crivelli SE, Denzler R, Stoffel M, Hall J. Miravirsen (SPC3649) can inhibit the biogenesis of miR-122. Nucleic Acids Res. 2014; 42: 609-21.

12. Titze-de-Almeida R, David C, Titze-de-Almeida SS. The Race of 10 Synthetic RNAi-Based Drugs to the Pharmaceutical Market. Pharmaceutical research. 2017; 34: 1339-63.

13. Li Z, Wong KY, Calin GA, Chng WJ, Chan GC, Chim CS. Epigenetic silencing of miR-340-5p in multiple myeloma: mechanisms and prognostic impact. Clinical epigenetics. 2019; 11: 71.

14. Novosadova E, Chabronova A, Kolek V, Petrek M, Navratilova Z. The Serum Expression of Selected miRNAs in Pulmonary Sarcoidosis 
with/without Lofgren's Syndrome. Mediators Inflamm. 2016; 2016: 1246129.

15. Smith-Vikos T, Liu Z, Parsons C, Gorospe M, Ferrucci L, Gill TM, et al. A serum miRNA profile of human longevity: findings from the Baltimore Longitudinal Study of Aging (BLSA). Aging (Albany NY). 2016; 8: 2971-87.

16. Zhou J, Gao J, Zhang X, Liu Y, Gu S, Zhang X, et al. microRNA-340-5p Functions Downstream of Cardiotrophin-1 to Regulate Cardiac Eccentric Hypertrophy and Heart Failure via Target Gene Dystrophin. Int Heart J. 2015; 56: 454-8

17. Ma $Y$, Shan $Z$, Ma J, Wang $Q$, Chu J, Xu P, et al. Validation of downregulated microRNAs during osteoclast formation and osteoporosis progression. Molecular medicine reports. 2016; 13: 2273-80.

18. Yu J, Wang R, Chen J, Wu J, Dang Z, Zhang Q, et al. miR-340 Inhibits Proliferation and Induces Apoptosis in Gastric Cancer Cell Line SGC-7901, Possibly via the AKT Pathway. Medical science monitor : international medical journal of experimental and clinical research. 2017; 23: 71-7.

19. Xiao C, Hong H, Yu H, Yuan J, Guo C, Cao H, et al. MiR-340 affects gastric cancer cell proliferation, cycle, and apoptosis through regulating SOCS3/JAK-STAT signaling pathway. Immunopharmacology and immunotoxicology. 2018; 40: 278-83.

20. Huang Z, Li Q, Luo K, Zhang Q, Geng J, Zhou X, et al. miR-340-FHL2 axis inhibits cell growth and metastasis in ovarian cancer. Cell Death Dis. 2019; 10: 372.

21. Klutstein M, Nejman D, Greenfield R, Cedar H. DNA Methylation in Cancer and Aging. Cancer research. 2016; 76: 3446-50.

22. Schmid G, Notaro S, Reimer D, Abdel-Azim S, Duggan-Peer M, Holly J, et al. Expression and promotor hypermethylation of miR-34a in the various histological subtypes of ovarian cancer. BMC Cancer. 2016; 16: 102.

23. Ning X, Shi Z, Liu X, Zhang A, Han L, Jiang K, et al. DNMT1 and EZH2 mediated methylation silences the microRNA-200b/a/429 gene and promotes tumor progression. Cancer Lett. 2015; 359: 198-205.

24. Corney DC, Hwang CI, Matoso A, Vogt M, Flesken-Nikitin A, Godwin AK, et al. Frequent downregulation of miR-34 family in human ovarian cancers. Clin Cancer Res. 2010; 16: 1119-28.

25. Das S, Bryan K, Buckley PG, Piskareva O, Bray IM, Foley N, et al. Modulation of neuroblastoma disease pathogenesis by an extensive network of epigenetically regulated microRNAs. Oncogene. 2013; 32: 2927-36.

26. Hashimoto Y, Akiyama Y, Yuasa Y. Multiple-to-multiple relationships between microRNAs and target genes in gastric cancer. PLoS One. 2013; 8: e62589.

27. Li XJ, Ren ZJ, Tang JH. MicroRNA-34a: a potential therapeutic target in human cancer. Cell Death Dis. 2014; 5: e1327.

28. Yamakuchi M, Lowenstein CJ. MiR-34, SIRT1 and p53: the feedback loop. Cell Cycle. 2009; 8: 712-5.

29. Hou LK, Yu Y, Xie YG, Wang J, Mao JF, Zhang B, et al. miR-340 and ZEB1 negative feedback loop regulates TGF-beta- mediated breast cancer progression. Oncotarget. 2016; 7: 26016-26.

30. Zhang J, Zhang Q, Lou Y, Fu Q, Chen Q, Wei T, et al. Hypoxia-inducible factor-1alpha/interleukin-1beta signaling enhances hepatoma epithelial-mesenchymal transition through macrophages in a hypoxic-inflammatory microenvironment. Hepatology. 2018; 67: 1872-89.

31. Geng H, Xue C, Mendonca J, Sun XX, Liu Q, Reardon PN, et al. Interplay between hypoxia and androgen controls a metabolic switch conferring resistance to androgen/AR-targeted therapy. Nature communications. 2018; 9: 4972 .

32. Bandara KV, Michael MZ, Gleadle JM. MicroRNA Biogenesis in Hypoxia. Microrna. 2017; 6: 80-96.

33. Nijhuis A, Thompson H, Adam J, Parker A, Gammon L, Lewis A, et al. Remodelling of microRNAs in colorectal cancer by hypoxia alters metabolism profiles and 5-fluorouracil resistance. Hum Mol Genet. 2017; 26: 1552-64.

34. Wozniak M, Sztiller-Sikorska M, Czyz M. Diminution of miR-340-5p levels is responsible for increased expression of ABCB5 in melanoma cells under oxygen-deprived conditions. Experimental and molecular pathology. 2015; 99: 707-16

35. Du K, Li Z, Fang X, Cao T, Xu Y. Ferulic acid promotes osteogenesis of bone marrow-derived mesenchymal stem cells by inhibiting microRNA-340 to induce beta-catenin expression through hypoxia. Eur J Cell Biol. 2017; 96: 496-503.

36. Yang D, Li Y, Zhao D. Curcumin induces apoptotic cell death in human pancreatic cancer cells via the miR-340/XIAP signaling pathway. Oncol Lett. 2017; 14: 1811-6.

37. Dolati S, Aghebati-Maleki L, Ahmadi M, Marofi F, Babaloo Z, Ayramloo $\mathrm{H}$, et al. Nanocurcumin restores aberrant miRNA expression profile in multiple sclerosis, randomized, double-blind, placebo-controlled trial. Journal of cellular physiology. 2018; 233: 5222-30.

38. Han X, Liu CF, Gao N, Zhao J, Xu J. Kaempferol suppresses proliferation but increases apoptosis and autophagy by up-regulating microRNA-340 in human lung cancer cells. Biomed Pharmacother. 2018; 108: 809-16.

39. Yan J, Qin Y, Yu J, Peng Q, Chen X. MiR-340/iASPP axis affects UVB-mediated retinal pigment epithelium (RPE) cell damage. J Photochem Photobiol B. 2018; 186: 9-16.

40. Qin Y, Zhou X, Huang C, Li L, Liu H, Liang N, et al. Lower miR-340 expression predicts poor prognosis of non-small cell lung cancer and promotes cell proliferation by targeting CDK4. Gene. 2018; 675: 278-84.

41. Yin G, Zhou H, Xue Y, Yao B, Zhao W. MicroRNA-340 promotes the tumor growth of human gastric cancer by inhibiting cyclin G2. Oncol Rep. 2016; 36: 1111-8.

42. Li X, Gong X, Chen J, Zhang J, Sun J, Guo M. miR-340 inhibits glioblastoma cell proliferation by suppressing CDK6, cyclin-D1 and cyclin-D2. Biochemical and biophysical research communications. 2015; 460: 670-7.

43. Polyak K, Lee MH, Erdjument-Bromage H, Koff A, Roberts JM, Tempst $\mathrm{P}$, et al. Cloning of p27Kip1, a cyclin-dependent kinase inhibitor and a potential mediator of extracellular antimitogenic signals. Cell. 1994; 78: 59-66.

44. Fernandez S, Risolino M, Mandia N, Talotta F, Soini Y, Incoronato M, et al. miR-340 inhibits tumor cell proliferation and induces apoptosis by targeting multiple negative regulators of p27 in non-small cell lung cancer. Oncogene. 2015; 34: 3240-50

45. Xue Y, Meehan B, Fu Z, Wang XQD, Fiset PO, Rieker R, et al. SMARCA4 loss is synthetic lethal with CDK4/6 inhibition in non-small cell lung cancer. Nature communications. 2019; 10: 557

46. Yu W, Zhang G, Lu B, Li J, Wu Z, Ma H, et al. MiR-340 impedes the progression of laryngeal squamous cell carcinoma by targeting EZH2. Gene. 2016; 577: 193-201.

47. Wang Z, Song D, Huang P. MicroRNA340 inhibits tumor cell proliferation, migration and invasion, and induces apoptosis in hepatocellular carcinoma. Molecular medicine reports. 2017; 16: 7649-56.

48. Yuan J, Ji H, Xiao F, Lin Z, Zhao X, Wang Z, et al. MicroRNA-340 inhibits the proliferation and invasion of hepatocellular carcinoma cells by targeting JAK1. Biochemical and biophysical research communications. 2017; 483: 578-84.

49. Sun Y, Zhao X, Zhou Y, Hu Y. miR-124, miR-137 and miR-340 regulate colorectal cancer growth via inhibition of the Warburg effect. Oncology reports. 2012; 28: 1346-52

50. Zhang LL, Xie FJ, Tang $\mathrm{CH}, \mathrm{Xu}$ WR, Ding XS, Liang J. miR-340 suppresses tumor growth and enhances chemosensitivity of colorectal cancer by targeting RLIP76. Eur Rev Med Pharmacol Sci. 2017; 21: 2875-86.

51. Wang $\mathrm{X}$, Song $\mathrm{Y}$. MicroRNA-340 inhibits the growth and invasion of angiosarcoma cells by targeting SIRT7. Biomed Pharmacother. 2018; 103: 1061-8.

52. Huang D, Qiu S, Ge R, He L, Li M, Li Y, et al. miR-340 suppresses glioblastoma multiforme. Oncotarget. 2015; 6: 9257-70.

53. Kim S, Choi JY, Seok HJ, Park MJ, Chung HY, Bae IH. miR-340-5p Suppresses Aggressiveness in Glioblastoma Multiforme by Targeting Bcl-w and Sox2. Molecular therapy Nucleic acids. 2019; 17: 245-55.

54. Pan BL, Wu L, Pan L, Yang YX, Li HH, Dai YJ, et al. Up-regulation of microRNA-340 promotes osteosarcoma cell apoptosis while suppressing proliferation, migration, and invasion by inactivating the CTNNB1-mediated Notch signaling pathway. Biosci Rep. 2018; 38.

55. Yan S, Jiang H, Fang S, Yin F, Wang Z, Jia Y, et al. MicroRNA-340 Inhibits Esophageal Cancer Cell Growth and Invasion by Targeting Phosphoserine Aminotransferase 1. Cellular physiology and biochemistry : international journal of experimental cellular physiology, biochemistry, and pharmacology. 2015; 37: 375-86.

56. Shi S, Chen X, Liu H, Yu K, Bao Y, Chai J, et al. LGR5 acts as a target of miR-340-5p in the suppression of cell progression and drug resistance in breast cancer via Wnt/beta-catenin pathway. Gene. 2019; 683: 47-53.

57. Huang K, Tang Y, He L, Dai Y. MicroRNA-340 inhibits prostate cancer cell proliferation and metastasis by targeting the MDM2-p53 pathway. Oncology reports. 2016; 35: 887-95.

58. Zhao P, Ma W, Hu Z, Zhang Y, Zhang S, Wang Y. Up-regulation of $\mathrm{miR}-340-5 \mathrm{p}$ promotes progression of thyroid cancer by inhibiting BMP4. Journal of endocrinological investigation. 2018; 41: 1165-72.

59. Song SZ, Lin S, Liu JN, Zhang MB, Du YT, Zhang DD, et al. Targeting of SPP1 by microRNA-340 inhibits gastric cancer cell epithelialmesenchymal transition through inhibition of the PI3K/AKT signaling pathway. Journal of cellular physiology. 2019; 234: 18587-601

60. Hassan M, Watari H, AbuAlmaaty A, Ohba Y, Sakuragi N. Apoptosis and molecular targeting therapy in cancer. Biomed Res Int. 2014; 2014: 150845 . 
61. Yigit S, Demir L, Tarhan MO, Cabuk FK, Ellidokuz H, Erten C, et al. The clinicopathological significance of Bax and Bcl-2 protein expression with tumor infiltrating lymphocytes in ovarian carcinoma. Neoplasma. 2012; 59: $475-85$.

62. Stylianou S, Clarke RB, Brennan K. Aberrant activation of notch signaling in human breast cancer. Cancer research. 2006; 66: 1517-25.

63. Shi L, Chen J, Yang J, Pan T, Zhang S, Wang Z. MiR-21 protected human glioblastoma U87MG cells from chemotherapeutic drug temozolomide induced apoptosis by decreasing Bax/Bcl-2 ratio and caspase- 3 activity. Brain Res. 2010; 1352: 255-64.

64. Arivazhagan R, Lee J, Bayarsaikhan D, Kwak P, Son M, Byun K, et al. MicroRNA-340 inhibits the proliferation and promotes the apoptosis of colon cancer cells by modulating REV3L. Oncotarget. 2018; 9: 5155-68.

65. Xie W, Qin W, Kang Y, Zhou Z, Qin A. MicroRNA-340 Inhibits Tumor Cell Proliferation and Induces Apoptosis in Endometrial Carcinoma Cell Line RL 95-2. Medical science monitor : international medical journal of experimental and clinical research. 2016; 22: 1540-6.

66. Li P, Sun Y, Liu Q. MicroRNA-340 Induces Apoptosis and Inhibits Metastasis of Ovarian Cancer Cells by Inactivation of NF-кB1. Cellular physiology and biochemistry : international journal of experimental cellular physiology, biochemistry, and pharmacology. 2016; 38: 1915-27.

67. Qu F, Wang X. microRNA-340 induces apoptosis by downregulation of BAG3 in ovarian cancer SKOV3 cells. Die Pharmazie. 2017; 72: 482-6.

68. Singh M, Yelle N, Venugopal C, Singh SK. EMT: Mechanisms and therapeutic implications. Pharmacol Ther. 2018; 182: 80-94.

69. Wu ZS, Wu Q, Wang CQ, Wang XN, Wang Y, Zhao JJ, et al. MiR-339-5p inhibits breast cancer cell migration and invasion in vitro and may be a potential biomarker for breast cancer prognosis. BMC Cancer. 2010; 10: 542.

70. Wu ZS, Wu Q, Wang CQ, Wang XN, Huang J, Zhao JJ, et al. miR-340 inhibition of breast cancer cell migration and invasion through targeting of oncoprotein c-Met. Cancer. 2011; 117: 2842-52.

71. Mohammadi-Yeganeh S, Paryan M, Arefian E, Vasei M, Ghanbarian H, Mahdian R, et al. MicroRNA-340 inhibits the migration, invasion, and metastasis of breast cancer cells by targeting Wnt pathway. Tumour biology : the journal of the International Society for Oncodevelopmental Biology and Medicine. 2016; 37: 8993-9000.

72. Chen CP, Sun ZL, Lu X, Wu WX, Guo WL, Lu JJ, et al. MiR-340 suppresses cell migration and invasion by targeting MYO10 in breast cancer. Oncology reports. 2016; 35: 709-16.

73. Yu Y, Xiao CH, Tan LD, Wang QS, Li XQ, Feng YM. Cancer-associated fibroblasts induce epithelial-mesenchymal transition of breast cancer cells through paracrine TGF-beta signalling. British journal of cancer. 2014; 110: 724-32

74. Xiao H, Yu L, Li F, Wang H, Li W, He X. MiR-340 suppresses the metastasis by targeting EphA3 in cervical cancer. Cell Biol Int. 2018

75. Trepo C, Chan HL, Lok A. Hepatitis B virus infection. Lancet. 2014; 384: 2053-63.

76. Xiong Q, Wu S, Wang J, Zeng X, Chen J, Wei M, et al. Hepatitis B virus promotes cancer cell migration by downregulating miR-340-5p expression to induce STAT3 overexpression. Cell Biosci. 2017; 7: 16.

77. Zhou X, Wei M, Wang W. MicroRNA-340 suppresses osteosarcoma tumor growth and metastasis by directly targeting ROCK1. Biochemical and biophysical research communications. 2013; 437: 653-8.

78. Takeyama H, Yamamoto H, Yamashita S, Wu X, Takahashi H, Nishimura J, et al. Decreased miR-340 expression in bone marrow is associated with liver metastasis of colorectal cancer. Molecular cancer therapeutics. 2014; 13: 976-85

79. Wang N, Xiang X, Chen K, Liu P, Zhu A. Targeting of NT5E by miR-30b and miR-340 attenuates proliferation, invasion and migration of gallbladder carcinoma. Biochimie. 2018; 146: 56-67.

80. Xu H, Lin F, Wang Z, Yang L, Meng J, Ou Z, et al. CXCR2 promotes breast cancer metastasis and chemoresistance via suppression of AKT1 and activation of COX2. Cancer Lett. 2018; 412: 69-80.

81. Cai W, Chen G, Luo Q, Liu J, Guo X, Zhang T, et al. PMP22 Regulates Self-Renewal and Chemoresistance of Gastric Cancer Cells. Molecular cancer therapeutics. 2017; 16: 1187-98.

82. Dasari S, Tchounwou PB. Cisplatin in cancer therapy: molecular mechanisms of action. European journal of pharmacology. 2014; 740: 364-78.

83. Pan JY, Zhang F, Sun CC, Li SJ, Li G, Gong FY, et al. miR-134: A Human Cancer Suppressor? Molecular therapy Nucleic acids. 2017; 6: 140-9.

84. Cai J, Fang L, Huang Y, Li R, Xu X, Hu Z, et al. Simultaneous overactivation of Wnt/beta-catenin and TGFbeta signalling by miR-128-3p confers chemoresistance-associated metastasis in NSCLC. Nature communications. 2017; 8: 15870

85. Yan H, Zhang B, Fang C, Chen L. miR-340 alleviates chemoresistance of osteosarcoma cells by targeting ZEB1. Anticancer Drugs. 2018; 29: 440-8.
86. Song L, Duan P, Gan Y, Li P, Zhao C, Xu J, et al. MicroRNA-340-5p modulates cisplatin resistance by targeting LPAATbeta in osteosarcoma. Brazilian journal of medical and biological research $=$ Revista brasileira de pesquisas medicas e biologicas. 2017; 50: e6359.

87. Shi L, Chen ZG, Wu LL, Zheng JJ, Yang JR, Chen XF, et al. miR-340 reverses cisplatin resistance of hepatocellular carcinoma cell lines by targeting Nrf2-dependent antioxidant pathway. Asian Pacific journal of cancer prevention : APJCP. 2014; 15: 10439-44.

88. Wu LL, Cai WP, Lei X, Shi KQ, Lin XY, Shi L. NRAL mediates cisplatin resistance in hepatocellular carcinoma via miR-340-5p/Nrf2 axis. J Cell Commun Signal. 2018

89. Rezaei Z, Sebzari A, Kordi-Tamandani DM, Dastjerdi K. Involvement of the Dysregulation of miR-23b-3p, miR-195-5p, miR-656-5p, and miR-340-5p in Trastuzumab Resistance of HER2-Positive Breast Cancer Cells and System Biology Approach to Predict Their Targets Involved in Resistance. DNA and cell biology. 2019; 38: 184-92.

90. Carter JV, Galbraith NJ, Yang D, Burton JF, Walker SP, Galandiuk S. Blood-based microRNAs as biomarkers for the diagnosis of colorectal cancer: a systematic review and meta-analysis. British journal of cancer. 2017; 116: 762-74.

91. Dong L, Li Y, Han C, Wang X, She L, Zhang H. miRNA microarray reveals specific expression in the peripheral blood of glioblastoma patients. International journal of oncology. 2014; 45: 746-56

92. Regazzo G, Terrenato I, Spagnuolo M, Carosi M, Cognetti G, Cicchillitti $\mathrm{L}$, et al. A restricted signature of serum miRNAs distinguishes glioblastoma from lower grade gliomas. Journal of experimental \& clinical cancer research : CR. 2016; 35: 124.

93. Guo J, Miao Y, Xiao B, Huan R, Jiang Z, Meng D, et al. Differential expression of microRNA species in human gastric cancer versus non-tumorous tissues. Journal of gastroenterology and hepatology. 2009; 24: 652-7.

94. Sueta A, Yamamoto Y, Tomiguchi M, Takeshita T, Yamamoto-Ibusuki M, Iwase H. Differential expression of exosomal miRNAs between breast cancer patients with and without recurrence. Oncotarget. 2017; 8: 69934-44

95. Fiore D, Donnarumma E, Roscigno G, Iaboni M, Russo V, Affinito A, et al. miR-340 predicts glioblastoma survival and modulates key cancer hallmarks through down-regulation of NRAS. Oncotarget. 2016; 7: 19531-47.

96. Liu Y, Li X, Zhang Y, Wang H, Rong X, Peng J, et al. An miR-340-5p-macrophage feedback loop modulates the progression and tumor microenvironment of glioblastoma multiforme. Oncogene. 2019; 38: 7399-415.

97. Pantel K, Alix-Panabieres C. Circulating tumour cells in cancer patients: challenges and perspectives. Trends Mol Med. 2010; 16: 398-406.

98. Flatmark K, Borgen E, Nesland JM, Rasmussen H, Johannessen HO, Bukholm I, et al. Disseminated tumour cells as a prognostic biomarker in colorectal cancer. British journal of cancer. 2011; 104: 1434-9.

99. Yang L, Men WL, Yan KM, Tie J, Nie YZ, Xiao HJ. MiR-340-5p is a potential prognostic indicator of colorectal cancer and modulates ANXA3. European review for medical and pharmacological sciences. 2018; 22: 4837-45.

100. Rath N, Olson MF. Rho-associated kinases in tumorigenesis: re-considering ROCK inhibition for cancer therapy. EMBO Rep. 2012; 13: 900-8.

101. Cai H, Lin L, Cai H, Tang M, Wang Z. Combined microRNA-340 and ROCK1 mRNA profiling predicts tumor progression and prognosis in pediatric osteosarcoma. International journal of molecular sciences. 2014; 15: 560-73.

102. Bian J, Liu R, Fan T, Liao L, Wang S, Geng W, et al. miR-340 Alleviates Psoriasis in Mice through Direct Targeting of IL-17A. J Immunol. 2018; 201: 1412-20.

103. Raychaudhuri M, Bronger H, Buchner T, Kiechle M, Weichert W, Avril S. MicroRNAs miR-7 and miR-340 predict response to neoadjuvant chemotherapy in breast cancer. Breast cancer research and treatment. 2017; 162: 511-21.

104. Cosset E, Petty T, Dutoit V, Tirefort D, Otten-Hernandez P, Farinelli L, et al. Human tissue engineering allows the identification of active miRNA regulators of glioblastoma aggressiveness. Biomaterials. 2016; 107: 74-87.

105. Xi Q, Zhang J, Yang G, Zhang L, Chen Y, Wang C, et al. Restoration of miR-340 controls pancreatic cancer cell CD47 expression to promote macrophage phagocytosis and enhance antitumor immunity. Journal for immunotherapy of cancer. 2020; 8.

106. Huang T, Zhou Y, Zhang J, Wong CC, Li W, Kwan JSH, et al. SRGAP1, a crucial target of miR-340 and miR-124, functions as a potential oncogene in gastric tumorigenesis. Oncogene. 2018; 37: 1159-74.

107. Hou X, Qiao H. Effect of miR-340 on gastric cancer cell proliferation and apoptosis. International journal of clinical and experimental pathology. 2015; 8: 13108-13. 
108. Song L, Zhou Z, Gan Y, Li P, Xu Y, Zhang Z, et al. Long noncoding RNA OIP5-AS1 causes cisplatin resistance in osteosarcoma through inducing the LPAATbeta/PI3K/AKT/mTOR signaling pathway by sponging the miR-340-5p. Journal of cellular biochemistry. 2019; 120: 9656-66.

109. Su P, Mu S, Wang Z. Long Noncoding RNA SNHG16 Promotes Osteosarcoma Cells Migration and Invasion via Sponging miRNA-340. DNA and cell biology. 2019; 38: 170-5.

110. Chen J, Lin $Y$, Jia Y, Xu T, Wu F, Jin Y. LncRNA HAND2-AS1 exerts anti-oncogenic effects on ovarian cancer via restoration of BCL2L11 as a sponge of microRNA-340-5p. Journal of cellular physiology. 2019.

111. Liu Y, Gao X, Tian X. High expression of long intergenic non-coding RNA LINC00662 contributes to malignant growth of acute myeloid leukemia cells by upregulating ROCK1 via sponging microRNA-340-5p. European journal of pharmacology. 2019; 859: 172535 .

112. Xie L, Chen Z, Liu H, Guan L, Wang Z, Li W. Effects of miR-340 on hepatocellular carcinoma by targeting the DcR3 gene. Digestive and liver disease : official journal of the Italian Society of Gastroenterology and the Italian Association for the Study of the Liver. 2018; 50: 291-6.

113. Poenitzsch Strong AM, Setaluri V, Spiegelman VS. MicroRNA-340 as a modulator of RAS-RAF-MAPK signaling in melanoma. Archives of biochemistry and biophysics. 2014; 563: 118-24.

114. Lu G, Zhang Y. MicroRNA-340-5p suppresses non-small cell lung cancer cell growth and metastasis by targeting ZNF503. Cellular \& molecular biology letters. 2019; 24: 34 .

115. Rongxin S, Pengfei L, Li S, Xiaochen J, Yihe H. MicroRNA-340-5p suppresses osteosarcoma development by down-regulating the Wnt/beta-catenin signaling pathway via targeting the STAT3 gene. European review for medical and pharmacological sciences. 2019; 23: 982-91.

116. Xu P, Li Y, Zhang H, Li M, Zhu H. MicroRNA-340 Mediates Metabolic Shift in Oral Squamous Cell Carcinoma by Targeting Glucose Transporter-1. Journal of oral and maxillofacial surgery : official journal of the American Association of Oral and Maxillofacial Surgeons. 2016; 74: 844-50.

117. Zhou Y, Li Z, Ding Y, Zhang P, Wang J. MicroRNA-340 suppresses pancreatic cancer growth by targeting BICD2. Pancreatology : official journal of the International Association of Pancreatology. 2019.

118. Mohammadi Yeganeh S, Vasei M, Tavakoli R, Kia V, Paryan M. The effect of miR-340 over-expression on cell-cycle-related genes in triple-negative breast cancer cells. European journal of cancer care. 2017; 